\title{
Learning about the structure of giant resonances from their $\gamma$ decay
}

\author{
W. L. Lv (吕万里) $\odot,{ }^{1}$ Y. F. Niu (牛一斐) $\odot,{ }^{1, *}$ and G. Colò $\odot^{2,3}$ \\ ${ }^{1}$ School of Nuclear Science and Technology, Lanzhou University, Lanzhou 730000, China \\ ${ }^{2}$ Dipartimento di Fisica, Unversità degli Studi di Milano, via Celoria 16, I-20133 Milano, Italy \\ ${ }^{3}$ INFN, Sezione di Milano, via Celoria 16, I-20133 Milano, Italy
}

(Received 23 July 2020; revised 6 May 2021; accepted 7 June 2021; published 17 June 2021)

\begin{abstract}
The direct $\gamma$ decays of the giant dipole resonance (GDR) and the giant quadrupole resonance (GQR) of ${ }^{208} \mathrm{~Pb}$ to low-lying states are investigated by means of a microscopic self-consistent model. The model considers effects beyond the linear response approximation. The experimental $\gamma$-decay widths from GQR to the first $3^{-}$state and the low-lying dipole states are well reproduced with the Skyrme interaction SkP. The strong sensitivity of $\gamma$ decay to the isospin of the involved states is proven. By comparing the decay widths between GDR to $2_{1}^{+}$and GQR to $3_{1}^{-}$, a much larger weight of the $3_{1}^{-}$component in the GQR wave function of ${ }^{208} \mathrm{~Pb}$ is deduced, with respect to the weight of the $2_{1}^{+}$component in the GDR wave function. Thus, we have shown that $\gamma$ decay is a unique probe of the resonance wave functions, and a test ground for nuclear structure models.
\end{abstract}

DOI: 10.1103/PhysRevC.103.064321

\section{INTRODUCTION}

Collective excitation modes of many-body systems exist in many branches of physics. In atomic nuclei the giant resonances (GRs) show up around 10-30 MeV [1-3], and they are characterized by the quantum numbers related to orbital angular momentum, spin, parity, and isospin. Since the discovery of the first GR mode, i.e., the giant dipole resonance (GDR) in 1937 [4], more and more GR modes have been discovered and studied. Giant resonances are not only interesting in themselves, but one should also recall that their properties can be linked to basic parameters of the nuclear equation of state (EoS) [5]. However, most of our well-established knowledge is still at the level of global properties, such as the mean resonance energy and the exhaustion of the appropriate energy-weighted sum rule (EWSR). Despite past attempts, a more detailed understanding of the wave functions of the GRs, as well as of their decay properties, is still missing to a large extent.

Giant resonances are characterized by a large decay width $\Gamma$ of several MeV. $\Gamma$ consists of several components. The first one is the Landau width $\Delta \Gamma$, resulting from the fragmentation of elementary one-particle-one hole (1p-1h) excitations, that do not all couple with themselves to form a single macroscopic collective state. The second one is the escape width $\Gamma^{\uparrow}$, related to the direct emission of nucleons, $\alpha$ particles, or photons. The last one is the spreading width $\Gamma^{\downarrow}$, that constitutes as a rule the dominant contribution to the total width and arises from the coupling to $2 \mathrm{p}-2 \mathrm{~h}, \ldots, n \mathrm{p}-n \mathrm{~h}$ states [6,7].

Although the above picture is widely accepted, the direct experimental evidences are not many. While the total width can be determined in inclusive experiments, there are only

*niuyf@1zu.edu.cn few decay experiments that have tried to pin down the precise values of $\Gamma^{\uparrow}$, and are even less able to extract the branching ratios $\Gamma_{c}^{\uparrow} / \Gamma^{\uparrow}$ associated to specific decay channels $c$ (cf. Table 8.3 of Ref. [3], as well as Ref. [8] and references therein). There have been several high-resolution experiments, but microscopic interpretations have been scarce. One exception is the series of experiments carried out by the Darmstadt group that have been complemented by a wavelet analysis of the underlying energy scales. The comparisons with the scales that emerged from theoretical models has allowed to draw a few tentative conclusions. It has been shown that, for the giant quadrupole resonance (GQR) in ${ }^{208} \mathrm{~Pb}$, the coupling of $1 \mathrm{p}-1 \mathrm{~h}$ configurations to the low-lying phonons is responsible for the fine structure [9], while for the GDR in ${ }^{208} \mathrm{~Pb}$ a different mechanism, i.e., Landau damping at the $1 \mathrm{p}-1 \mathrm{~h}$ level, leads to the same fine structure [10].

$\Gamma_{\gamma}$ is a tiny part of the total decay width, but since only the well-known electromagnetic interaction is involved, the results can be more clearly interpreted than all those in which the strong interaction is involved [11]. The direct decay branch ought to be clearly distinguished from the decay through the compound nucleus [12]. Then, the $\gamma$ decay is extremely sensitive to the multipolarities of GRs [13,14], and its study provides a useful way to shed light on the microscopic properties of the GRs. Although these ideas have been put forward in the past, the quantitative studies are also very rare. Some of them were made when nuclear structure models were purely phenomenological [15], so that it was hard to assess if $\gamma$ decay was a strong benchmark to test theories. Instead, here, we use Skyrme functionals self-consistently. Thus, the main goal of our work is to show quantitatively how the $\gamma$ decay can give access to microscopic properties of GRs and, in this respect, how it can be a test ground for nuclear models.

The experimental data on the $\gamma$ decay of GRs to low-lying states are very rare due to the the small branching ratio. A 
few data were reported several decades ago $[13,14]$; recently, new data were reported in Ref. [16]. However, with the development of new $\gamma$-beam facilities like ELI-NP in Europe [17] and SLEGS in China [18], as well as with the advancing of $\gamma$-ray detectors like ELIGANT-GN [19], the measurement of $\gamma$ decay to low-lying states becomes more and more feasible, so that new experiments are planned at ELI-NP [19] and RCNP [20].

Under such circumstances, the theoretical study on the GR decay to low-lying states needs a revival. Several models based on phenomenological inputs, including the surface coupling model [15], the perturbation theory within the nuclear field theory (NFT) formalism [21], the extended theory of finite Fermi systems (ETFFS) [22,23], and the quasiparticlephonon model (QPM) $[24,25]$, have been employed so far. All these models incorporate effects beyond the mean-field level.

Only a few years ago, the first fully self-consistent treatment of the $\gamma$ decay of GRs, based on the random phase approximation plus particle-vibration coupling (RPA+PVC) model, has become available [26], It has to be noted that the RPA+PVC model has been successfully applied to study different nuclear properties, ranging from the single-particle nuclear levels [27-30], the Gamow-Teller response and the related $\beta$ decay [31-35], to the spreading widths of noncharge-exchange GRs [36,37]. Therefore, in this work, we use the RPA+PVC model to investigate the $\gamma$ decay of GRs to low-lying states through electric dipole $(E 1)$ transitions and provide useful guidance for possible experiments in the future. The novelty of our work, with respect to previous ones, consists in having been able to interpret the theoretical results for the $\gamma$ decay in terms of basic properties of the involved states (in particular, their isospin), and in terms of their microscopic wave function.

\section{FORMALISM}

The $\gamma$-decay width $\Gamma_{\gamma}$ is calculated as [38]

$$
\Gamma_{\gamma}(E \lambda ; i \rightarrow f)=\frac{8 \pi(\lambda+1)}{\lambda[(2 \lambda+1) ! !]^{2}}\left(\frac{E}{\hbar c}\right)^{2 \lambda+1} B(E \lambda ; i \rightarrow f),
$$

where $E$ represents the transition energy, and $\lambda$ is the transition multipolarity. The transition probability $B$ is

$$
B(E \lambda ; i \rightarrow f)=\frac{1}{2 J_{i}+1}\left|\left\langle J_{f} \| Q_{\lambda}|| J_{i}\right\rangle\right|^{2},
$$

where the initial and final vibrational states $|n J\rangle$, denoted by a wavy line in Fig. 1, are calculated with the fully self-consistent RPA method [39]. In general, the electric multipole operator reads

$$
\begin{aligned}
Q_{\lambda \mu}= & \frac{e}{2} \sum_{i=1}^{A}\left\{\left[\left(1-\frac{1}{A}\right)^{\lambda}+(-)^{\lambda} \frac{2 Z-1}{A^{\lambda}}\right]\right. \\
& \left.-\left[\left(1-\frac{1}{A}\right)^{\lambda}+\frac{(-)^{\lambda+1}}{A^{\lambda}}\right] \tau_{z}(i)\right\} r_{i}^{\lambda} \mathrm{i}^{\lambda} Y_{\lambda \mu}\left(\hat{\boldsymbol{r}}_{i}\right) \\
\equiv & \frac{1}{2} \sum_{i=1}^{A} e_{i}^{\mathrm{eff}} r_{i}^{\lambda} \mathrm{i}^{\lambda} Y_{\lambda \mu}\left(\hat{\boldsymbol{r}}_{i}\right),
\end{aligned}
$$

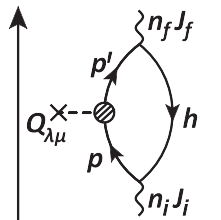

(A)

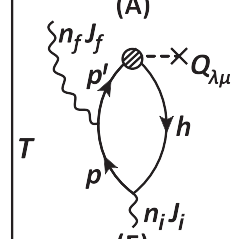

(E)
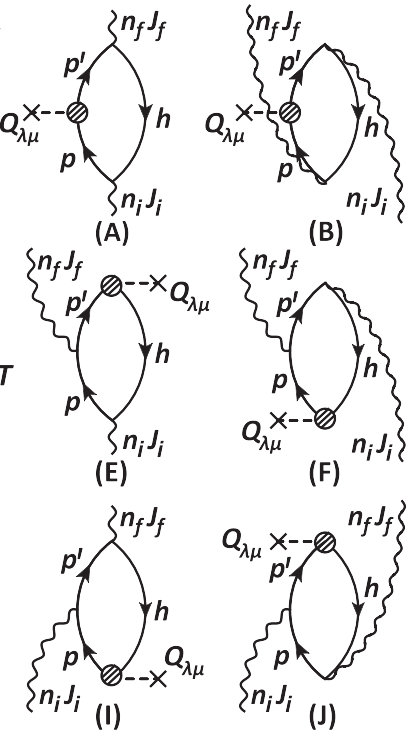

(B)

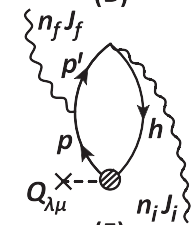

(F)

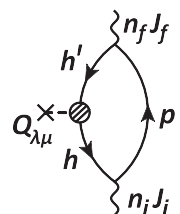

(C)

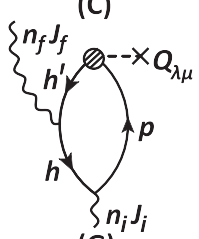

(G)

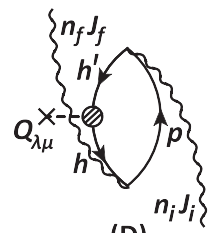

(D)

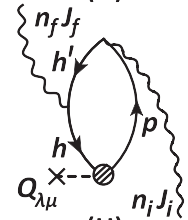

(H)
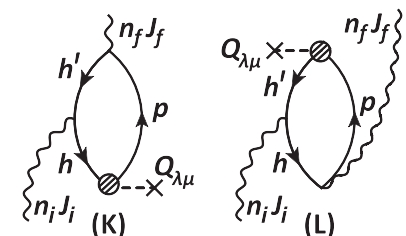

(J)

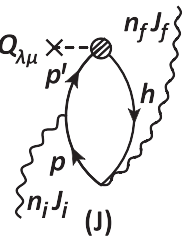

FIG. 1. The 12 lowest-order NFT diagrams in the process of $\gamma$ decay between two vibrational states. The circle with lines includes the contribution to $Q_{\lambda \mu}$ from nuclear polarization [26]. The arrow of time is upward.

where the effective charge caused by the recoil of the nucleus has been introduced [38].

For the decay between two vibrational states, the RPA method, which describes well only the transitions between states that differ only by one vibrational phonon, is not enough. We have to consider the interplay between collective phonons and individual particles, i.e., the particle-vibration coupling (PVC) effects. This can be dealt with in a perturbative approach, by including all the lowest-order perturbative diagrams involving single-particle states, phonons, and two particle-phonon vertices. This is analogous to what had been done within the framework of the NFT $[21,40,41]$. The singleparticle states are calculated in Hartree-Fock (HF), and the nuclear vibrations are built out of the single-particle degrees of freedom by means of a self-consistent RPA. The lowestorder diagrams that involve two PVC vertices are sketched in Fig. 1, and they are used in the calculation of the reduced matrix element $\left\langle J_{f}\left\|Q_{\lambda}\right\| J_{i}\right\rangle$. The model is the same as in Ref. [26], where more details can be found. Diagrams A-D are at the RPA level, while diagrams E-L are at the PVC level due to the PVC vertex, e.g., $\left\langle p^{\prime}, n J\left|V_{\text {res. }}\right| p\right\rangle$ in diagram E. Diagrams E, F, G, and $\mathrm{H}$ will contribute when the initial phonon has more complex configurations made up with $1 \mathrm{p}-1 \mathrm{~h}$ coupled with the final phonon, while diagrams I, J, $\mathrm{K}$, and $\mathrm{L}$ will contribute when the final phonon has more complex configurations made up with $1 \mathrm{p}-1 \mathrm{~h}$ coupled with the initial phonon. The interaction $V_{\text {res. }}$ at the particle-holephonon vertex is the residual two-body interaction, which is obtained self-consistently from the Skyrme energy functional by $V_{\text {res. }}^{m i, n j}=\frac{\delta^{2} E_{\mathrm{HF}}}{\delta \rho_{m i} \delta \rho_{n j}}$.

\section{RESULTS AND DISCUSSIONS}

The present calculation is done in a configuration space where the cutoff energy for single-particle levels is $E_{\text {cut }}=$ 
TABLE I. Energies $E$ and electric transition probabilities $B(E \lambda)$ of the first $2^{+}$and $3^{-}$states, as well as centroid energies and electric transition probabilities $B(E \lambda)$ of the GDR and GQR in ${ }^{208} \mathrm{~Pb}$. The experimental values are from Refs. [42,43].

\begin{tabular}{|c|c|c|c|c|c|c|c|c|}
\hline Expt. & 4.09 & 2.62 & $13.5 \pm 0.1$ & $10.9 \pm 0.3$ & $3.18 \pm 0.16$ & $6.11 \pm 0.09$ & $62.5 \pm 5.0$ & $5.80 \pm 1.60$ \\
\hline $\mathrm{SkM}^{*}$ & 4.86 & 3.22 & 13.65 & 11.63 & 2.91 & 5.77 & 73.2 & 5.00 \\
\hline SkP & 3.64 & 3.12 & 13.49 & 10.18 & 2.77 & 5.76 & 66.9 & 5.96 \\
\hline
\end{tabular}

$150 \mathrm{MeV}$, which is much larger than that used in Ref. [26] $\left(E_{\text {cut }}=50 \mathrm{MeV}\right)$, and thus reaches converged results. The box size for calculating the single-particle levels is $20 \mathrm{fm}$. The EWSRs satisfy the double commutator values at the level of more than $99.50 \%$. Giant resonance is known to be a broad structure that is spread over an energy region of several $\mathrm{MeV}$. In our study, the total decay width of GRs is the sum of all widths from those RPA states with energies in the range 10-18 MeV and a fraction of either isoscalar (IS) or isovector (IV) EWSR that is larger than 5\%. The decay width is sensitive to the properties of the initial state $\left|n_{i} J_{i}\right\rangle$ and final state $\left|n_{f} J_{f}\right\rangle$. Therefore, as a first step, we check the quality of the description of the low-lying states $2_{1}^{+}$and $3_{1}^{-}$, as well as that of the GDR and GQR, by comparing energies and electric transition probabilities with the experimental values. These latter are listed in Table I together with the theoretical results obtained using four of the many Skyrme sets, namely, LNS [44], SkM* [45], SkP [46], and SLy5 [47]. Among these four interactions, SkP describes well both the energies and electric transition probabilities for low-lying states and GRs, since the largest discrepancy with experimental data is less than $20 \%$. In particular, for the energies $E$ and $B(E \lambda)$ values of GRs, the discrepancies are not larger than $10 \%$. Moreover, the calculated $B(E 1 \uparrow)_{\mathrm{GDR}}$ and $B(E 2 \uparrow)_{\mathrm{GQR}}$ lie within the experimental error.

In Table II, we list the $\gamma$-decay widths $\Gamma_{\gamma}$ or relative branching ratio $\Gamma_{\gamma} / \Gamma_{\gamma}^{\mathrm{g} . s .}$ of GRs to low-lying states, as well as the total width of GRs. For the total widths of GRs, the last two lines in Table II, we follow the RPA+PVC model used in Ref. [49], where the phonons coupling with particle-hole configurations are considered as intermediate virtual states. These intermediate states are with $J^{\pi}=0^{+}, 1^{-}, 2^{+}, 3^{-}, 4^{+}$, energies smaller than $20 \mathrm{MeV}$, and fraction of sum rule (IS or IV) $\frac{B_{i}(\mathrm{IS})}{\sum B_{i}(\mathrm{IS})}$ or $\frac{B_{i}(\mathrm{IV})}{\sum B_{i}(\mathrm{IV})}>5 \%$. One can see that the Skyrme interaction SkP can well reproduce both the $\gamma$-decay widths and total widths, including the decay widths of the ISGQR to the $3_{1}^{-}$state and to the low-lying dipole states lying between 5 and $7 \mathrm{MeV}$. In Ref. [13], except for the decay from ISGQR to $3_{1}^{-}$, other transitions from the ISGQR to low-lying states were also studied, and a good agreement with experimental data has been obtained with the QPM model [24]. As listed in Table II, we also calculated the decay width of the ISGQR to the low-lying dipole states lying between 5 and $7 \mathrm{MeV}$, and a decay branch relative to ground state of 0.97 is obtained, showing a good agreement with experimental data, $1.15 \pm 0.5$ [13]. However, our model cannot reproduce the strong dipole transition to the $3^{-}$state at $4.97 \mathrm{MeV}$, being similar as earlier work within the NFT formalism [21]. We note that in Ref. [13] the experimental relative branching ratio of the $\gamma$ decay of GQR to the $2^{+}(4.085 \mathrm{MeV})$ state has also been reported as small as $0.02_{-0.02}^{+0.05}$, which is not considered in our calculation.

Based on the results in Tables I and II, it is reliable to use SkP in the following investigation of the $\gamma$ decay from GDR $(\mathrm{GQR})$ to the $2_{1}^{+}\left(3_{1}^{-}\right)$state. Before we go a step further we try to see if some properties of the interactions can be associated with a better or worse performance in the reproduction of the decay widths. This is not obvious a priori. While giant resonance energies can be associated with bulk properties of the Skyrme forces, this may not be true for their decay. Still, we can attempt some speculation, related mainly to the effective

TABLE II. The $\gamma$-decay width $\Gamma_{\gamma}$ and relative $\gamma$-branching ratio $\Gamma_{\gamma} / \Gamma_{\gamma}^{\text {g.s. }}$ of GRs to low-lying states. The total width of GRs are also listed here. The experimental values are from Refs. [13,48].

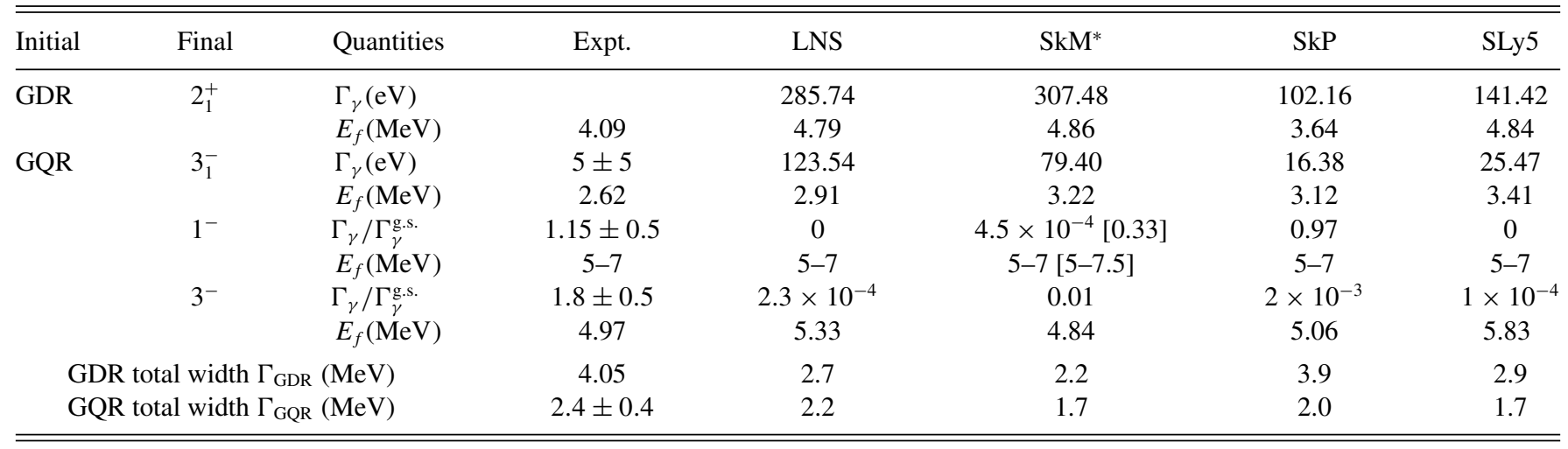


mass, as this governs the overall level density. Among the four Skyrme functionals, i.e., SkP, LNS, SkM*, and SLy5, the isoscalar effective mass $m_{s}^{*} / m$ of SkP is the largest, as 1.0. This could be the reason for its good quantitative description of the $\gamma$-decay widths from GQR, which is reasonable since the large isoscalar effective mass $m_{s}^{*} / m$ is also desirable to reproduce the peak energies of the isoscalar monopole and quadrupole modes, seeing the most recent study on the quadrupole modes in Mo isotopes [50]. As for the $\gamma$ decay from GDR to the $2_{1}^{+}$state, due to the lack of experimental data, it is hard to evaluate the Skyrme functionals. However, we still noticed that the $\gamma$-decay width $\Gamma_{\gamma}\left(\mathrm{GDR} \rightarrow 2_{1}^{+}\right)$could be correlated with the slope of symmetry energy $L$ and isovector effective mass $m_{v}^{*} / m . \Gamma_{\gamma}\left(\mathrm{GDR} \rightarrow 2_{1}^{+}\right)$of these four Skyrme functionals are very different, showing that this decay is a sensitive probe for isovector properties of nuclear interaction. On the other hand, the centroid energy of GDR can be well reproduced by all four functionals, which could result from their similar isovector effective mass $m_{v}^{*} / m$ [51,52]. A more robust statistical analysis may be needed in future to confirm or disprove these speculations. More experimental data on the $\gamma$ decay of GDR would be helpful to evaluate the Skyrme functionals and to constrain the properties of nuclear matter.

Then we use SkP to investigate the properties of the $\gamma$-decay widths from GRs to low-lying states. Although the qualities of different Skyrme interactions in the present context vary, by checking the results of all four Skyrme interactions, we found that the main conclusions of this paper are independent of the choice of the interaction, including the isovector (isoscalar) property of the GDR (GQR) state, and the larger amount of the low-lying $33_{1}^{-}$phonon coupling component in the wave function of GQR than that of the $2_{1}^{+}$ phonon coupling component in the wave function of GDR.

For the $\gamma$-decay widths $\Gamma_{\gamma}$ calculated by SkP, $\Gamma_{\gamma}(\mathrm{GDR} \rightarrow$ $\left.2_{1}^{+}\right)$is $102.16 \mathrm{eV}$, larger than six times of $\Gamma_{\gamma}\left(\mathrm{GQR} \rightarrow 3_{1}^{-}\right)$, which is $16.38 \mathrm{eV}$. Notice that the averaged transition energies for these two cases are respectively 9.85 and $7.06 \mathrm{MeV}$, which cause only two to three times difference in the decay widths. To understand this large difference of decay widths, we first notice that the isospin properties of GDR and GQR are quite different, as the GDR (GQR) is mainly an isovector (isoscalar) resonance. Therefore, we consider separately the contributions from protons $(\pi)$ and neutrons $(\nu)$ to the total $\gamma$-decay widths, that are shown in Fig. 2(a). One can see that for the GDR, the total decay width $\Gamma_{\gamma}$ is larger than the decay widths stemming from only protons $\left(\Gamma_{\gamma}^{\pi}\right)$ or neutrons $\left(\Gamma_{\gamma}^{\nu}\right)$, that is, $\Gamma_{\gamma}>\Gamma_{\gamma}^{\pi(v)}$. For the GQR, on the contrary, $\Gamma_{\gamma}<\Gamma_{\gamma}^{\pi(v)}$. This means that protons and neutrons contribute coherently to enhance the $\gamma$-decay width in the transition GDR $\rightarrow 2_{1}^{+}$, while they cancel each other to reduce the $\gamma$-decay width in the transition GQR $\rightarrow 3_{1}^{-}$. This behavior can be explained by considering that the low-lying states $2_{1}^{+}$and $3_{1}^{-}$have both isoscalar character and that the $E 1$ operator is isovector. As a result, under the action of the $E 1$ operator, the proton and neutron contributions have the same phase in the GDR $\rightarrow 2_{1}^{+}$ case, but opposite phase for the GQR $\rightarrow 3_{1}^{-}$case, so that these decays are, respectively, either enhanced or suppressed $[15,53]$. Such isospin dependence of $\gamma$ decay was also analyzed in detail in Ref. [24] by using the quasiparticle-phonon

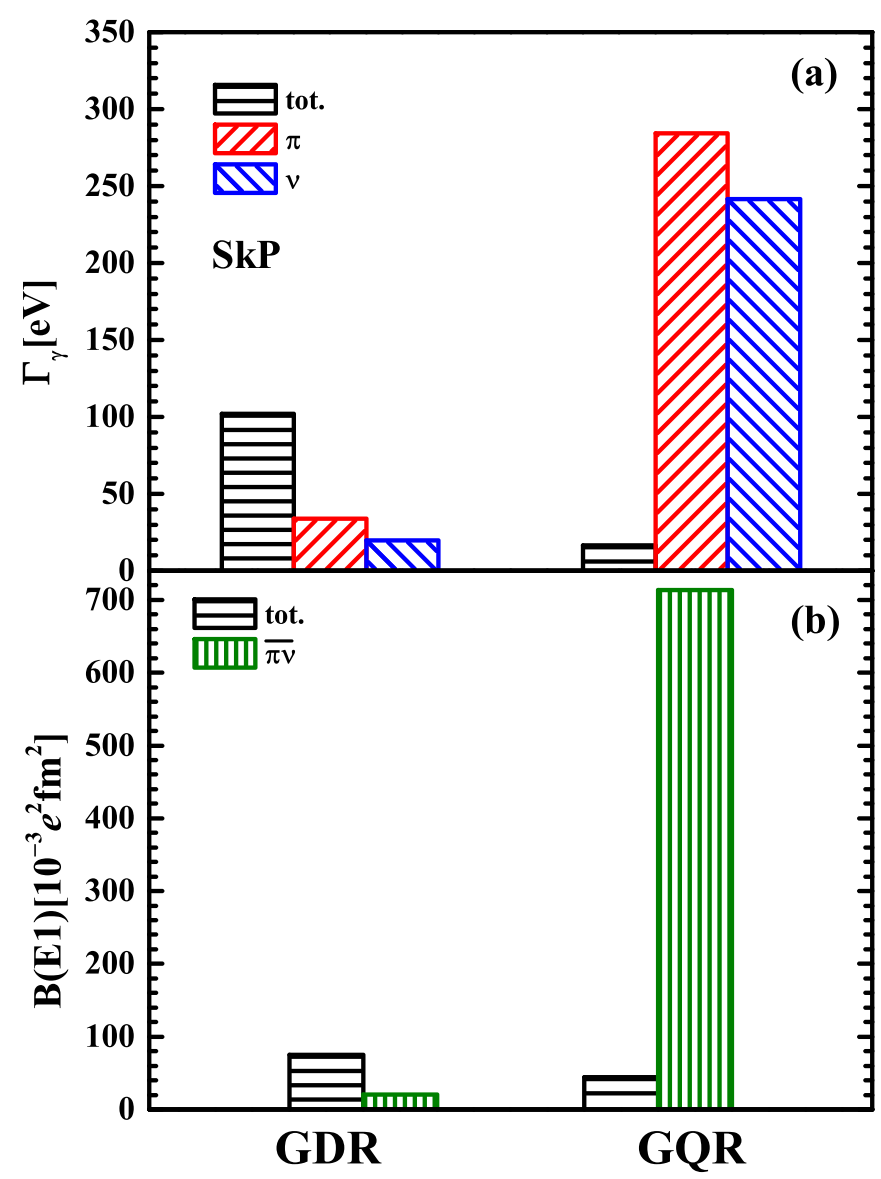

FIG. 2. (a) Comparison of the $\gamma$-decay widths between GDR $\rightarrow$ $2_{1}^{+}$and GQR $\rightarrow 3_{1}^{-}$in ${ }^{208} \mathrm{~Pb}$. The total $\gamma$-decay widths (tot.) are shown, as well as the contributions from protons $(\pi)$ and neutrons (v). (b) Comparison of the transition probabilities $B(E 1)$ between $\mathrm{GDR} \rightarrow 2_{1}^{+}$and GQR $\rightarrow 3_{1}^{-}$in ${ }^{208} \mathrm{~Pb}$. Here, the total $B(E 1)$ and the average of contributions from protons and neutrons $B^{\overline{\pi v}}(E 1)$ are displayed.

model. The authors of Ref. [24] have compared the values of $\Gamma_{\gamma}$ from the ISGQR and IVGQR to the $3_{1}^{-}$state in ${ }^{208} \mathrm{~Pb}$ : the decay width of the ISGQR to the $3_{1}^{-}$state is very small, while the decay width of the IVGQR is practically the same as that associated to the transition to the ground state, for the same reason explained above. Thus, this result is consistent with our conclusions.

We can further prove the above argument in the limit of exact isospin symmetry. We pick up the $N=Z$ nucleus ${ }^{56} \mathrm{Ni}$ and turn off the Coulomb interaction. In this case, the $2_{1}^{+}$and $3_{1}^{-}$ states are purely isoscalar, while the GDR and GQR states are purely isovector and isoscalar, respectively. The contributions to the $\gamma$-decay widths from protons and neutrons, $\Gamma_{\gamma}^{\pi}$ and $\Gamma_{\gamma}^{\nu}$, are equal. In this limit, a neat relation is found, namely,

$$
\begin{aligned}
\Gamma\left(\mathrm{GDR} \rightarrow 2_{1}^{+}\right) & =4 \Gamma^{\pi(v)}\left(\mathrm{GDR} \rightarrow 2_{1}^{+}\right), \\
\Gamma\left(\mathrm{GQR} \rightarrow 3_{1}^{-}\right) & =\Gamma^{\pi}\left(\mathrm{GQR} \rightarrow 3_{1}^{-}\right)-\Gamma^{\nu}\left(\mathrm{GQR} \rightarrow 3_{1}^{-}\right) \\
& =0 .
\end{aligned}
$$

In Table III, we show the results of our microscopic calculations that fully confirm the above expectation, of the largest 
TABLE III. Total $\gamma$-decay widths $\Gamma_{\gamma}$ and contributions from protons $\left(\Gamma_{\gamma}^{\pi}\right)$ and neutrons $\left(\Gamma_{\gamma}^{\nu}\right)$ for GDR $\rightarrow 2_{1}^{+}$and GQR $\rightarrow 3_{1}^{-}$, respectively, in ${ }^{56} \mathrm{Ni}$ without the Coulomb interaction. Units are in $\mathrm{eV}$.

\begin{tabular}{lrrr}
\hline \hline${ }^{56} \mathrm{Ni}$ & \multicolumn{1}{c}{$\Gamma_{\gamma}$} & \multicolumn{1}{c}{$\Gamma_{\gamma}^{\pi}$} & \multicolumn{1}{c}{$\Gamma_{\gamma}^{v}$} \\
\hline $\mathrm{GDR} \rightarrow 2_{1}^{+}$ & 3071.60 & 767.90 & 767.90 \\
$\mathrm{GQR} \rightarrow 3_{1}^{-}$ & 0.00 & 53.99 & 53.99 \\
\hline \hline
\end{tabular}

possible coherence between neutrons and protons in the GDR decay and of their complete cancellation in the GQR decay. This serves as a limiting example to which the situation in ${ }^{208} \mathrm{~Pb}$ may be compared. For the $\gamma$ decay of GQR $\rightarrow 3_{1}^{-}$in the neutron excess nucleus ${ }^{208} \mathrm{~Pb}$, the isospin selection rule holds well, both in the experimental and theoretical results. This indicates that the "effective" or "macroscopic" isospin symmetry in the collective mode is unaffected up to the first order in $(N-Z) / A$. The microscopic results are in harmony with early findings [41] without the need of imposing any constraint.

However, once we exclude the influence of the different isospin properties of GRs, and only compare $\Gamma_{\gamma}^{\pi}$ or $\Gamma_{\gamma}^{\nu}$ in the two cases, GDR $\rightarrow 2_{1}^{+}$and GQR $\rightarrow 3_{1}^{-}$in ${ }^{208} \mathrm{~Pb}$, a completely different pattern is observed. Specifically, $\Gamma_{\gamma}^{\pi}$ and $\Gamma_{\gamma}^{\nu}$ in the decay of the GDR are, respectively, 33.94 and $19.73 \mathrm{eV}$, while in the decay of the GQR they are both around ten times larger, namely, 284.31 and $242.08 \mathrm{eV}$.

To understand this relation, we first rule out the influence of the transition energies $E$ that enters $\Gamma_{\gamma}$, and we directly use the total transition probability $B(E 1)$ as well as the values of $B^{\overline{\pi v}}(E 1)$, that is, the average of the contributions from protons and neutrons [see Fig. 2(b)]. Similar relations as for the $\gamma$ decay widths are found for $B(E 1): B(E 1)$ for the GDR decay is larger than that for the GQR decay but, nevertheless, for $B^{\overline{\pi v}}(E 1)$ the value for the GDR decay is drastically less than $\frac{1}{30}$ of the value for the GQR decay:

$$
\begin{aligned}
B\left(E 1 ; \mathrm{GDR} \rightarrow 2_{1}^{+}\right)>B\left(E 1 ; \mathrm{GQR} \rightarrow 3_{1}^{-}\right), \\
B^{\overline{\pi v}}\left(E 1 ; \mathrm{GDR} \rightarrow 2_{1}^{+}\right)<\frac{1}{30} B^{\overline{\pi v}}\left(E 1 ; \mathrm{GQR} \rightarrow 3_{1}^{-}\right) .
\end{aligned}
$$

The latter of these two relations must have to do with the wave functions of the initial and final phonon.

As already mentioned, the $B(E 1)$ from the GRs to low-lying states obtains contributions from 12 different amplitudes. The associated diagrams are sensitive to different components of the wave function of the initial or final phonon.

In Fig. 3, we show the cumulative $\gamma$-decay widths $\Gamma_{\gamma}$ of the 12 diagrams to the $\gamma$-decay widths $\Gamma_{\gamma}$ of GDR $\rightarrow 2_{1}^{+}$ [Fig. 3(a)] and GQR $\rightarrow 3_{1}^{-}$[Fig. 3(b)]. In the case of the GDR decay [Fig. 3(a)], diagram A, representing the particle contribution at the RPA level, and diagram $\mathrm{C}$, representing the hole contribution at the same level, almost cancel each other; hence, only a small contribution remains at the RPA level (at which the wave function of both phonons is a superposition simply of $1 \mathrm{p}-1 \mathrm{~h}$ configurations). Once diagram $\mathrm{E}$, representing one of the particle contributions at the PVC

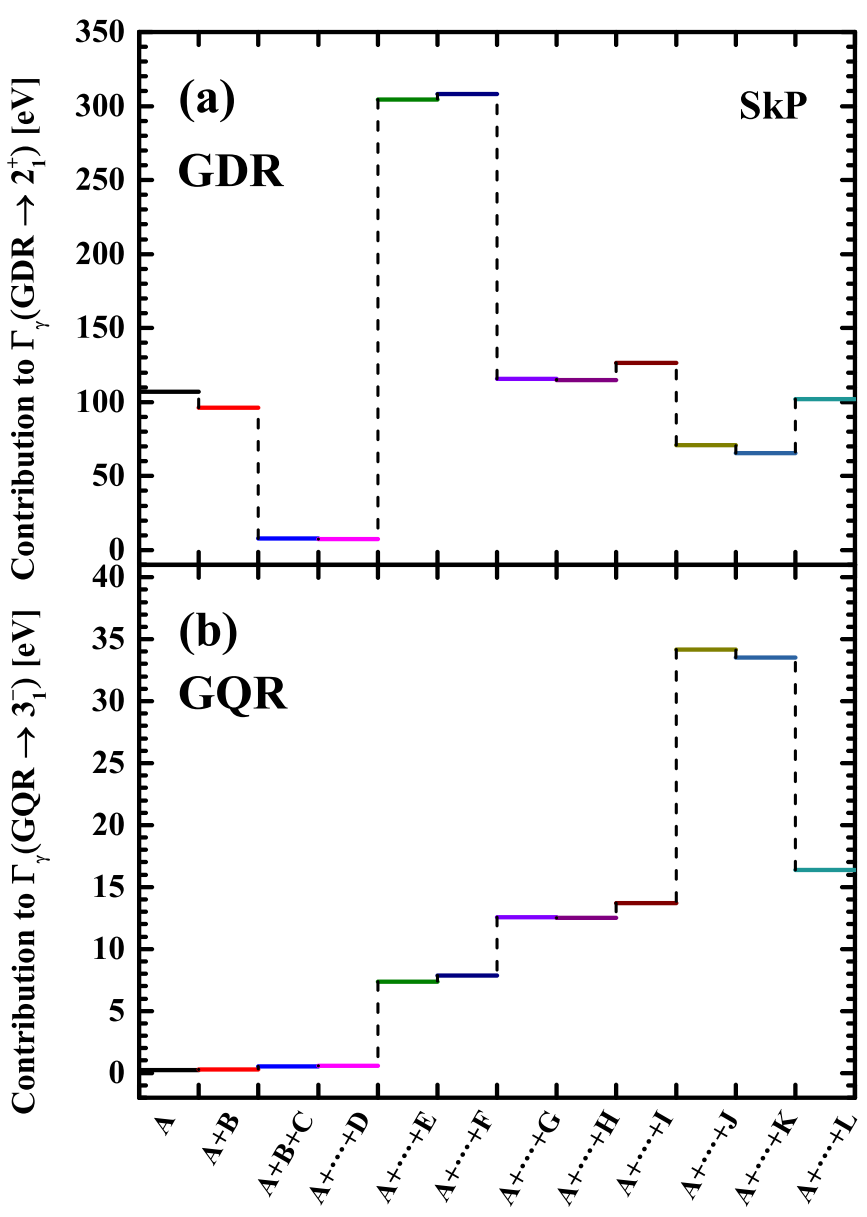

FIG. 3. The cumulative $\gamma$-decay widths $\Gamma_{\gamma}$ of 12 NFT diagrams of (a) GDR $\rightarrow 2_{1}^{+}$and (b) GQR $\rightarrow 3_{1}^{-}$in ${ }^{208} \mathrm{~Pb}$.

level, is considered, the width becomes very large; diagram $\mathrm{G}$, representing the corresponding hole contribution at the PVC level, cancels around $62 \%$ of the width that becomes $115.65 \mathrm{eV}$. Diagrams E and G represent the contributions (at PVC level) that arise when the wave function of the initial phonon has the component $\left|\left[(p h)_{J} \otimes J_{f}\right]_{J_{i}}\right\rangle$, namely, the coupling of $1 \mathrm{p}-1 \mathrm{~h}$ configurations with the final phonon. The contributions from the diagrams I and $\mathrm{K}$ are small: these diagrams represent the contributions (at the PVC level) that arise when the wave function of the final phonon has the component $\left|\left[(p h)_{J} \otimes J_{i}\right]_{J_{f}}\right\rangle$, which corresponds to the coupling of $1 \mathrm{p}-1 \mathrm{~h}$ configurations with the initial phonon. It is understandable that low-lying states can be coupled with $1 \mathrm{p}-1 \mathrm{~h}$ configurations and be admixed with GRs that lie at similar energies. Having a high-lying GR plus a $1 \mathrm{p}-1 \mathrm{~h}$ state in the wave function of a low-lying state is far more unlikely. In the end, diagrams $\mathrm{E}$ and $\mathrm{G}$ yield $84 \%$ of the total width.

For the GQR decay case in Fig. 3(b), all the diagrams at the RPA level are extremely small. When diagram $\mathrm{E}$ is taken into account, $\Gamma_{\gamma}$ is $7.37 \mathrm{eV}$. When diagram $\mathrm{G}$ is added, it becomes $12.55 \mathrm{eV}$. Diagram J contributes much to the width, but diagram L almost cancels it. Finally, we get $16.38 \mathrm{eV}$. Similar to the GDR case, the decay width calculated with only diagrams $\mathrm{E}$ and $\mathrm{G}$ is $17.19 \mathrm{eV}$, which is very close to 
TABLE IV. Contribution of each NFT diagram to $\gamma$-decay width $\Gamma_{\gamma}$. For each diagram, the contributions from protons and neutrons are distinguished. Units are in $\mathrm{eV}$.

\begin{tabular}{lrrrrrrr}
\hline \hline & \multicolumn{3}{c}{$\mathrm{GDR} \rightarrow 2_{1}^{+}$} & & \multicolumn{3}{c}{$\mathrm{GQR} \rightarrow 3_{1}^{-}$} \\
\cline { 2 - 4 } \cline { 7 - 9 } Diagram & \multicolumn{1}{c}{$\Gamma_{\gamma}^{\pi}$} & \multicolumn{1}{c}{$\Gamma_{\gamma}^{\nu}$} & \multicolumn{1}{c}{$\Gamma_{\gamma}^{\text {tot. }}$} & & $\Gamma_{\gamma}^{\pi}$ & $\Gamma_{\gamma}^{v}$ & $\Gamma_{\gamma}^{\text {tot. }}$ \\
\hline $\mathrm{A}$ & 48.61 & 16.73 & 106.78 & & 23.23 & 22.32 & 0.23 \\
$\mathrm{~B}$ & 0.12 & 0.05 & 0.32 & & 0.35 & 0.23 & 0.01 \\
$\mathrm{C}$ & 47.73 & 17.43 & 117.66 & & 23.70 & 16.85 & 0.58 \\
$\mathrm{D}$ & 0.08 & 0.03 & 0.21 & & 0.18 & 0.13 & 0.01 \\
$\mathrm{E}$ & 100.51 & 51.38 & 259.99 & & 173.36 & 171.53 & 10.13 \\
$\mathrm{~F}$ & 0.03 & 0.01 & 0.08 & & 0.22 & 0.15 & 0.01 \\
$\mathrm{G}$ & 24.78 & 8.74 & 48.53 & & 0.50 & 3.60 & 3.00 \\
$\mathrm{H}$ & 0.01 & $<0.01$ & 0.01 & & 0.02 & 0.02 & $<0.01$ \\
$\mathrm{I}$ & 0.57 & 0.22 & 1.46 & & 4.07 & 3.39 & 0.03 \\
$\mathrm{~J}$ & 3.85 & 1.71 & 8.87 & & 197.72 & 173.42 & 4.79 \\
$\mathrm{~K}$ & 0.32 & 0.16 & 0.90 & 2.75 & 2.53 & 0.01 \\
$\mathrm{~L}$ & 3.49 & 0.74 & 4.36 & & 111.31 & 97.32 & 3.21 \\
\hline \hline
\end{tabular}

the result with all 12 diagrams. We note that, at variance with the GDR decay case in Fig. 3(a), diagrams $\mathrm{J}$ and $\mathrm{L}$ give big contributions in the case of GQR $\rightarrow 3_{1}^{-}$. Diagrams $\mathrm{J}$ and $\mathrm{L}$ represent the contributions from the backward amplitude $Y_{p h}$ of the final phonon. Usually the backward amplitudes of RPA phonons are small; however, for the $3_{1}^{-}$state in ${ }^{208} \mathrm{~Pb}$, the $Y_{p h}$ amplitudes are large, and this leads to big values for diagrams $\mathrm{J}$ and $\mathrm{L}$.

In Table IV, we list the contribution of each NFT diagram to the $\gamma$-decay width $\Gamma_{\gamma}$, and the contributions from protons and neutrons are distinguished. First, the isospin dependence of $\gamma$ decay can be seen in each diagram. For the case of GDR $\rightarrow 2_{1}^{+}$, the total decay width of each diagram is the result of the coherent contribution of protons and neutrons, while for GQR $\rightarrow 3_{1}^{-}$, it results from the cancellation between protons and neutrons. Second, both $\Gamma_{\gamma}^{\pi}$ and $\Gamma_{\gamma}^{\nu}$ of GDR and GQR calculated by diagrams at the PVC level (E in the GDR case, and $\mathrm{E}, \mathrm{J}$, and $\mathrm{L}$ in the GQR case) are much larger than those calculated by diagrams at the RPA level. Although in the case of GQR $\rightarrow 3_{1}^{-}, \Gamma_{\gamma}^{\pi}$ and $\Gamma_{\gamma}^{\nu}$ calculated by diagram J or $\mathrm{L}$ are large, the cancellation between protons and neutrons is also prominent, so $\Gamma_{\gamma}^{\text {tot. }}$ of diagram $\mathrm{J}$ or $\mathrm{L}$ is much smaller.

As a conclusion, for both the GDR and GQR decay, diagrams $\mathrm{E}$ and $\mathrm{G}$ dominate the decay width. It means that in this study of $\gamma$ decay, the component of $1 \mathrm{p}-1 \mathrm{~h}$ coupled with the final phonon in the wave function of the initial phonon plays an essential role. Now, from the discovery that $B^{\overline{\pi v}}(E 1)$ of GQR $\rightarrow 3_{1}^{-}$is larger than that of GDR $\rightarrow 2_{1}^{+}$, as stated in Eq. (6b), we can conclude that the $\left|\left[(p h)_{J} \otimes 3_{1}^{-}\right]_{\mathrm{GQR}}\right\rangle$ component in the wave function of the GQR is much larger than the $\left|\left[(p h)_{J} \otimes 2_{1}^{+}\right]_{\mathrm{GDR}}\right\rangle$ component in the wave function of the GDR. This conclusion is in agreement with that of the wavelet analysis, that we mentioned in the first part of this paper. Nevertheless, as we have already stressed, we have demonstrated that the analysis of the $\gamma$ decay to low-lying vibrational states provides a more clear and direct way to draw this conclusion.

\section{SUMMARY}

In summary, the $\gamma$ decays from GRs to low-lying states in ${ }^{208} \mathrm{~Pb}$ are studied with the RPA+PVC model by calculating the lowest-order NFT diagrams. First, we have proven the strong sensitivity of $\gamma$ decay to the isospin of the involved states. Indeed, the decay GDR $\rightarrow 2_{1}^{+}$is isospin enhanced while the decay GQR $\rightarrow 3_{1}^{-}$is isospin suppressed. If we exclude the isospin effects and consider the proton-neutron average of the transition probabilities, $B^{\overline{\pi v}}(E 1)$, this is found to be two times larger in the case of GQR $\rightarrow 3_{1}^{-}$than in the case of GDR $\rightarrow 2_{1}^{+}$. This points clearly to a larger weight of the $\left|\left[(p h)_{J} \otimes 3_{1}^{-}\right]_{\mathrm{GQR}}\right\rangle$ component in the GQR wave function with respect to the $\left|\left[(p h)_{J} \otimes 2_{1}^{+}\right]_{\mathrm{GDR}}\right\rangle$ component in the GDR wave function.

This work shows explicitly that the $\gamma$ decay of GRs to low-lying vibrational states is an effective approach to access directly the microscopic structure of the GRs. The same kind of study can be extended to other cases of interest like, for instance, that of the so-called pygmy resonances (PRs) in order to understand to which extent they have a specific nature that makes them different from GRs. In general, we look forward to new experimental data for $\gamma$ decay, to answer more directly and systematically the questions what are the microscopic structure and damping mechanism of GRs and PRs, what are their isospin properties, how collective a PR is, and all the like.

\section{ACKNOWLEDGMENTS}

This research is supported by the National Natural Science Foundation of China under Grant No. 12075104, the Fundamental Research Funds for the Central Universities under Grants No. lzujbky-2021-it10 and No. lzujbky-2019-11, and the European Union's Horizon 2020 research and innovation program under Grant No. 654002.
[1] P. F. Bortignon, A. Bracco, and R. A. Broglia, Giant Resonances: Nuclear Structure at Finite Temperature (Harwood Academic, New York, 1998).

[2] J. Speth and A. van der Woude, Giant resonances in nuclei, Rep. Prog. Phys. 44, 719 (1981).

[3] M. N. Harakeh and A. van der Woude, Giant Resonances: Fundamental High-Frequency Modes of Nuclear Excitation (Oxford University Press, Oxford, 2001).
[4] W. Bothe and W. Gentner, Atomumwandlungen durch-strahlen, Z. Phys. 106, 236 (1937).

[5] X. Roca-Maza and N. Paar, Nuclear equation of state from ground and collective excited state properties of nuclei, Prog. Part. Nucl. Phys. 101, 96 (2018).

[6] G. F. Bertsch, P. F. Bortignon, and R. A. Broglia, Damping of nuclear excitations, Rev. Mod. Phys. 55, 287 (1983). 
[7] S. Drozdz, S. Nishizaki, J. Speth, and J. Wambach, The nuclear response within extended RPA theories, Phys. Rep. 197, 1 (1990).

[8] M. Hunyadi, H. Hashimoto, T. Li, H. Akimune, H. Fujimura, M. Fujiwara, Z. Gácsi, U. Garg, K. Hara, M. N. Harakeh, J. Hoffman, M. Itoh, T. Murakami, K. Nakanishi, B. K. Nayak, S. Okumura, H. Sakaguchi, S. Terashima, M. Uchida, Y. Yasuda, and M. Yosoi, Proton decay from the isoscalar giant dipole resonance in ${ }^{58} \mathrm{Ni}$, Phys. Rev. C 80, 044317 (2009).

[9] A. Shevchenko, J. Carter, R. W. Fearick, S. V. Förtsch, H. Fujita, Y. Fujita, Y. Kalmykov, D. Lacroix, J. J. Lawrie, P. von Neumann-Cosel, R. Neveling, V. Y. Ponomarev, A. Richter, E. Sideras-Haddad, F. D. Smit, and J. Wambach, Fine Structure in the Energy Region of the Isoscalar Giant Quadrupole Resonance: Characteristic Scales from a Wavelet Analysis, Phys. Rev. Lett. 93, 122501 (2004).

[10] I. Poltoratska, R. W. Fearick, A. M. Krumbholz, E. Litvinova, H. Matsubara, P. von Neumann-Cosel, V. Y. Ponomarev, A. Richter, and A. Tamii, Fine structure of the isovector giant dipole resonance in ${ }^{208} \mathrm{~Pb}$ : Characteristic scales and level densities, Phys. Rev. C 89, 054322 (2014).

[11] A. Bracco, F. Camera, F. C. L. Crespi, B. Million, and O. Wieland, Gamma-decay from dipole vibrations: Probe for nuclear properties, Eur. Phys. J. A 55, 233 (2019).

[12] J. Beene, G. Bertsch, P. Bortignon, and R. Broglia, Direct and compound gamma decay of the giant quadrupole resonance of ${ }^{208} \mathrm{~Pb}$, Phys. Lett. B 164, 19 (1985).

[13] J. R. Beene, F. E. Bertrand, M. L. Halbert, R. L. Auble, D. C. Hensley, D. J. Horen, R. L. Robinson, R. O. Sayer, and T. P. Sjoreen, Heavy-ion excitation and photon decay of giant resonances in ${ }^{208} \mathrm{~Pb}$, Phys. Rev. C 39, 1307 (1989).

[14] J. R. Beene, F. E. Bertrand, D. J. Horen, R. L. Auble, B. L. Burks, J. Gomez del Campo, M. L. Halbert, R. O. Sayer, W. Mittig, Y. Schutz, J. Barrette, N. Alamanos, F. Auger, B. Fernandez, A. Gillibert, B. Haas, and J. P. Vivien, Heavy-ion coulomb excitation and photon decay of the giant dipole resonance in ${ }^{208} \mathrm{~Pb}$, Phys. Rev. C 41, 920 (1990).

[15] P. F. Bortignon, R. A. Broglia, and G. F. Bertsch, Gamma decay of the giant quadrupole resonance in ${ }^{208} \mathrm{~Pb}$, Phys. Lett. B 148, 20 (1984).

[16] J. Isaak, D. Savran, M. Krtička, M. W. Ahmed, J. Beller, E. Fiori, J. Glorius, J. H. Kelley, B. Löher, N. Pietralla, C. Romig, G. Rusev, M. Scheck, L. Schnorrenberger, J. Silva, K. Sonnabend, A. P. Tonchev, W. Tornow, H. R. Weller, and M. Zweidinger, Constraining nuclear photon strength functions by the decay properties of photo-excited states, Phys. Lett. B 727, 361 (2013).

[17] K. A. Tanaka, K. M. Spohr, D. L. Balabanski, S. Balascuta, L. Capponi, M. O. Cernaianu, M. Cuciuc, A. Cucoanes, I. Dancus, A. Dhal, B. Diaconescu, D. Doria, P. Ghenuche, D. G. Ghita, S. Kisyov, V. Nastasa, J. F. Ong, F. Rotaru, D. Sangwan, P.-A. Söderström, D. Stutman, G. Suliman, O. Tesileanu, L. Tudor, N. Tsoneva, C. A. Ur, D. Ursescu, and N. V. Zamfir, Current status and highlights of the ELI-NP research program, Matter Radiat. Extremes 5, 024402 (2020).

[18] W. Guo, Y. Xu, J.-G. Chen, Y.-G. Ma, W. Xu, X.-Z. Cai, and H.-W. Wang, Shanghai laser electron gamma source and its applications, Chin. Phys. C 32(Suppl. II), 190 (2008).

[19] M. Krzysiek, F. Camera, D. Filipescu, H. Utsunomiya, G. Colò, I. Gheorghe, and Y. Niu, Simulation of the ELIGANT-GN array performances at ELI-NP for gamma beam energies larger than neutron threshold, Nucl. Instrum. Methods Phys. Res. Sect. A 916, 257 (2019).

[20] A. Bracco, P. von Neumann-Cosel, and A. Tamii, Gamma decay from giant resonances: A pilot experiment, RCNP proposal E498 (unpublished).

[21] D. R. Bes, P. Curutchet, S. L. Reich, N. N. Scoccola, and H. M. Sofia, Electromagnetic decay of the giant quadrupole resonances: II. Nuclear structure aspects, Nucl. Phys. A 452, 531 (1986).

[22] J. Speth, D. Cha, V. Klemt, and J. Wambach, Signature to detect the isovector giant quadrupole resonance, Phys. Rev. C 31, 2310 (1985).

[23] S. Kamerdzhiev, J. Speth, and G. Tertychny, Extended theory of finite Fermi systems: Collective vibrations in closed shell nuclei, Phys. Rep. 393, 1 (2004).

[24] V. Voronov and V. Ponomarev, On gamma-decay of giant resonances, Nucl. Phys. A 520, c619 (1990).

[25] V. Ponomarev and A. Krasznahorkay, On gamma decay of giant dipole resonance in tin isotopes, Nucl. Phys. A 550, 150 (1992).

[26] M. Brenna, G. Colò, and P. F. Bortignon, Microscopic theory of the $\gamma$ decay of nuclear giant resonances, Phys. Rev. C 85, 014305 (2012).

[27] E. Litvinova and P. Ring, Covariant theory of particlevibrational coupling and its effect on the single-particle spectrum, Phys. Rev. C 73, 044328 (2006).

[28] G. Colò, H. Sagawa, and P. F. Bortignon, Effect of particlevibration coupling on single-particle states: A consistent study within the Skyrme framework, Phys. Rev. C 82, 064307 (2010).

[29] E. V. Litvinova and A. V. Afanasjev, Dynamics of nuclear single-particle structure in covariant theory of particle-vibration coupling: From light to superheavy nuclei, Phys. Rev. C 84, 014305 (2011).

[30] G. Colò, P. F. Bortignon, and G. Bocchi, Hybrid configuration mixing model for odd nuclei, Phys. Rev. C 95, 034303 (2017).

[31] Y. F. Niu, G. Colò, and E. Vigezzi, Gamow-Teller response and its spreading mechanism in doubly magic nuclei, Phys. Rev. C 90, 054328 (2014).

[32] Y. F. Niu, Z. M. Niu, G. Colò, and E. Vigezzi, Particle-Vibration Coupling Effect on the $\beta$ Decay of Magic Nuclei, Phys. Rev. Lett. 114, 142501 (2015).

[33] Y. Niu, Z. Niu, G. Colò, and E. Vigezzi, Interplay of quasiparticle-vibration coupling and pairing correlations on $\gamma$ decay half-lives, Phys. Lett. B 780, 325 (2018).

[34] C. Robin and E. Litvinova, Time-Reversed Particle-Vibration Loops and Nuclear Gamow-Teller Response, Phys. Rev. Lett. 123, 202501 (2019).

[35] E. Litvinova, C. Robin, and H. Wibowo, Temperature dependence of nuclear spin-isospin response and beta decay in hot astrophysical environments, Phys. Lett. B 800, 135134 (2020)

[36] X. Roca-Maza, Y. F. Niu, G. Colò, and P. F. Bortignon, Towards a self-consistent dynamical nuclear model, J. Phys. G: Nucl. Part. Phys. 44, 044001 (2017).

[37] S. Shen, G. Colò, and X. Roca-Maza, Particle-vibration coupling for giant resonances beyond the diagonal approximation, Phys. Rev. C 101, 044316 (2020).

[38] A. Bohr and B. R. Mottelson, Nuclear Structure, 2nd ed. (W. A. Benjamin, New York, 1998), Vol. I. 
[39] G. Colò, L. Cao, N. V. Giai, and L. Capelli, Self-consistent RPA calculations with Skyrme-type interactions: The skyrme_rpa program, Comput. Phys. Commun. 184, 142 (2013).

[40] P. Bortignon, R. Broglia, D. Bes, and R. Liotta, Nuclear field theory, Phys. Rep. 30, 305 (1977).

[41] A. Bohr and B. R. Mottelson, Nuclear Structure, 2nd ed. (W. A. Benjamin, New York, 1998), Vol. II.

[42] M. Martin, Nuclear data sheets for $A=208$, Nucl. Data Sheets 108, 1583 (2007).

[43] A. Veyssiere, H. Beil, R. Bergere, P. Carlos, and A. Lepretre, Photoneutron cross sections of ${ }^{208} \mathrm{~Pb}$ and ${ }^{197} \mathrm{Au}$, Nucl. Phys. A 159, 561 (1970).

[44] L. G. Cao, U. Lombardo, C. W. Shen, and N. V. Giai, From Brueckner approach to Skyrme-type energy density functional, Phys. Rev. C 73, 014313 (2006).

[45] J. Bartel, P. Quentin, M. Brack, C. Guet, and H.-B. Håkansson, Towards a better parametrisation of Skyrme-like effective forces: A critical study of the SkM force, Nucl. Phys. A 386, 79 (1982).

[46] J. Dobaczewski, H. Flocard, and J. Treiner, Hartree-FockBogolyubov description of nuclei near the neutron-drip line, Nucl. Phys. A 422, 103 (1984).

[47] E. Chabanat, R. Bonche, R. Haensel, J. Meyer, and R. Schaeffer, A Skyrme parametrization from subnuclear to neutron star densities. Part II. Nuclei far from stabilities, Nucl. Phys. A 635 , 231 (1998).

[48] F. E. Bertrand, G. R. Satchler, D. J. Horen, J. R. Wu, A. D. Bacher, G. T. Emery, W. P. Jones, D. W. Miller, and A. van der Woude, Giant multipole resonances from inelastic scattering of 152-MeV alpha particles, Phys. Rev. C 22, 1832 (1980).

[49] G. Colò, N. Van Giai, P. F. Bortignon, and R. A. Broglia, Escape and spreading properties of charge-exchange resonances in ${ }^{208}$ Bi, Phys. Rev. C 50, 1496 (1994).

[50] G. Colò, D. Gambacurta, W. Kleinig, J. Kvasil, V. O. Nesterenko, and A. Pastore, Isoscalar monopole and quadrupole modes in Mo isotopes: Microscopic analysis, Phys. Lett. B 811, 135940 (2020).

[51] P. Klüpfel, P.-G. Reinhard, T. J. Bürvenich, and J. A. Maruhn, Variations on a theme by Skyrme: A systematic study of adjustments of model parameters, Phys. Rev. C 79, 034310 (2009).

[52] I. Stetcu, A. Bulgac, P. Magierski, and K. J. Roche, Isovector giant dipole resonance from the $3 \mathrm{D}$ time-dependent density functional theory for superfluid nuclei, Phys. Rev. C 84, 051309(R) (2011).

[53] J. Speth, E. Werner, and W. Wild, Theory of finite Fermi systems and application to the lead region, Phys. Rep. 33, 127 (1977). 